versity of Toronto) have isolated a strain of virus able to transform normal CFU-Es in vitro as judged by their ability to differentiate without erythropoietin. This may be analogous to the in vitro transformation of avian bone marrow cells by avian erythroblastosis virus (AEV) (T. Graf, MaxPlanck-Institut, Tübingen).

A diversity of studies have evolved from the isolation of Friend erythroleukaemic cells (FLC) in vitro (C. Friend, Mount Sinai School of Medicine, New York). These cells are CFUEs arrested at the proerythroblast stage, which after treatment with dimethylsulphoxide (DMSO) synthesise haemoglobin and differentiate to orthochromatic erythroblasts and posibly further (Y. Ikawa, Cancer Research Foundation, Tokyo). Other erythroid changes follow: spectrin formation and loss of $\mathrm{H}-2$ surface antigen ( $H$. Eisen, University of Geneva) and accumulation of carbonic anhydrase (F. Ruddle, Yale University). New compounds, such as highly polar molecules and fatty acids (R. Rifkind and P. Marks, Columbia University, New York) also act as inducers; haem also can induce globin synthesis and may act synergistically with DMSO (J. Ross, McArdle Laboratory, Wisconsin; A. Beaudet, Baylor College, Houston). This induction of erythroid differentiation in FLC contrasts with the situation in AEVtransformed avian erythroid cells which do not differentiate or produce haemoglobin in response to classical inducers of FLC (Graf), although they accumulate globin RNA in the nucleus (A. Therwath, University of Paris).

Little is known concerning the mechanisms of action of the various inducing agents. Membrane function may be important, however. A. Bernstein (Ontario Cancer Institute, Toronto) found that local anaesthetics which increase membrane fluidity block haemoglobin production. This is consistent with other membrane changes including rapid immunological "capping” (N. Furusawa, Osaka City University), increased agglutination with plant lectins (Eisen), decreased permeability to small molecules (S. Dube, California Institute of Technology), increased anisotropy (D. Jovin, Max-Planck-Institut, Göttingen) and changes in cyclic nucleotides ( $R$. Kram and P. Malpoix, University of Brussels).

Regarding the cell cycle dependence of the induction process, haemoglobin is not detectable until cells have been exposed to DMSO for about two cell cycles (J. Papaconstantinou, Oak Ridge National Laboratory; Marks and Rifkind). However, J. Pragnell (Beatson Institute, Glasgow) and W. Ostertag (Max-Planck-Institut, Göttingen) reported that one FLC line shows a 5-fold increase in globin mRNA within the first cell doubling.

Several laboratories have attempted to elucidate the mechanisms regulating globin gene expression in FLC, exploiting DNA complementary to globin mRNA to measure synthesis and accumulation of globin-specific RNA. H. Aviv (Weizmann Institute, Rehovoth) stressed that relative rates of degradation of globin and total mRNAs are important in understanding how globin mRNAs predominate in the reticulocyte or differentiated FLC. Unfortunately even the sensitive techniques used are insufficient to permit similar analyses at the nuclear level to clarify the role of transcriptional and post-transcriptional regulation mechanisms. The fact that the base-sequence complexity of poly(A)-containing nuclear RNA is considerably greater than that of polysomal poly(A)-containing RNA indicates post-transcriptional control of gene activity (M. Birnie and J. Paul, Beatson Institute, Glasgow).

Another approach to regulatory mechanisms was described by $\mathbf{P}$. Harrison and D. Conkie (Beatson Institute) utilising non-inducible FLC variants. In some, non-inducibility of globin synthesis is due to a dominant

\section{Chemical and biological reactions}

IN my recent comments (Nature, 256, 693; 1975) on dynamics of chemical and biological reactions I mentioned work by Uzgiris and Golibersuch (Phys. Rev. Lett., 32, 37 ; 1974) attributing a decay term independent of scattering angle in scattered-light intensity fluctuations from haemoglobin solutions to the haemoglobin association-dissociation reaction. A paper by Haas, Mustacich, Smith and Ware (Biochem. biophys. Res. Commun., 59, 174; 1974) which disputes these findings has now come to my attention. These authors attribute the "anomalous" positive intercepts in the plot of linewidth versus the square of the scattering vector reported by other authors to the coincidence of the Helium-Neon wavelength and the $630 \mathrm{~nm}$ absorption of methaemoglobin. I am bringing these findings to the attention of your readers in view of the importance of establishing whether reaction rate of fast chemical and biological reaction can be derived from time autocorrelation functions.

I would also like to state that the fluorescence of ethidium bromide is strongly enhanced, and not quenched (as mentioned in my comments) upon binding to DNA. H. EISENBERG defect at the nuclear level (either in globin gene transcription or an unstable primary RNA transcript); whereas in another, treatment with haem enhances globin and globin mRNA accumulation. Interestingly, in a lymphoma $\times$ FLC hybrid, globin mRNA accumulates, but globin chains do not; nor does erythroid maturation occur unless the hybrid cells are treated with haem in addition to DMSO. A regulatory role for haem in differentiation of FLC is also implied by the report of $\mathrm{G}$. Kramer and $\mathbf{B}$. Hardesty (University of Austin, Texas) that there are two inhibitors of translation in Friend cells, one of which is present in DMSO-treated cells only and bears close similarity to the classical haemin-controlled repressor present in reticulocytes.

Others are also using cell fusion to analyse the regulation of differentiation. B. Alter (Childrens Hospital, Boston) showed that some human globins were synthesised in heterokaryons between tetraploid FLC and diploid human amniotic fibroblasts but not in hybrids with diploid FLC. A. Skoultchi (Albert Einstein, New York) observed that hybrids between FLC and mouse hepatoma cells continue to synthesise albumin and transferrin but cannot be induced to synthesise haemoglobin or globin mRNAs. The potential of somatic cell genetics for genetic mapping was illustrated by A. Deisseroth (National Institutes of Health) by exploiting stable mouse-human hybrid clones to correlate loss of human globin genes with loss of specific chromosomes. It should only be a matter of months before the location of the $\alpha$ and $\beta$ genes are known with certainty.

As regards the virus-host interactions, it is clear that at least three genes of the mouse determine the course of the erythroblastic disease induced by FV. The $F v-1$ locus controls the ability of the helper murine leukaemia virus (MLV) component to initiate productive infection. A second gene, $F v-2$, controls the animal's response to the spleen focus-forming element (SFFV) of FV; in a resistant mouse, MLV and SFFV replicate and although morphologically distinct spleen foci do not appear, splenomegaly occurs later ( 5 weeks) (T. Odaka, Institute of Medical Science, Tokyo). K. Blank (Albert Einstein, New York) showed the restrictive effect of each locus not only on the spleen focus formation but also on the abiliy of the animal to limit growth of the tumour colony-forming cells. A third locus, the major histocompatibility locus $(H-2)$, seems to affect not the initial infection by $\mathrm{FV}$, but rather the subsequent ability to support chronic infection (H. Freedman, Albert Einstein, 